\title{
Desempenho de diferentes guias de ondas para uso com o analisador de umidade TRASE
}

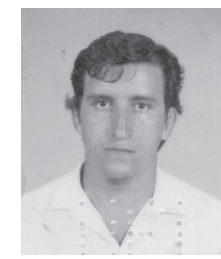

\author{
Eugênio F. Coelho ${ }^{1}$, Camilo de L.T. Andrade' ${ }^{2}$, Dani Or ${ }^{3}$, Lucy C. Lopes ${ }^{4}$ \& Claudinei F. Souza ${ }^{5}$
}

1 Embrapa Fruticultura Tropical. R. Embrapa s/n, C.P. 7, Cruz das Almas, CEP 44380-000, BA. E-mail: ecoelho@cnpmf.embrapa.br (Foto)
2 Embrapa - Milho e Sorgo. CEP $35700-000$, Sete Lagoas, MG
${ }^{3}$ Utah State University Logan, UT, USA, 84321-4820
${ }^{4}$ Bolsista CAPES - Cruz das Almas BA
${ }^{5}$ FEAGRI/UNICAMP, Departamento de Água e Solo. C.P. 6011 , CEP 13081-970, Campinas, SP. E-mail: cfsouza@agr.unicamp.br

Protocolo $114-04 / 09 / 2000$

\begin{abstract}
Resumo: O trabalho teve como objetivo avaliar a relação entre a umidade e a constante dielétrica aparente do solo e diferentes guias de onda para uso com o analisador de umidade Trase, que opera de acordo com o princípio da reflectometria no domínio do tempo - TDR. Amostras indeformadas e deformadas de duas manchas de textura diferente de um Latossolo Amarelo Distrófico foram retiradas do campo e acondicionadas em recipientes de $10 \mathrm{~L}$, perfazendo quatro repetições para cada textura e estrutura. Foram construídas três diferentes guias de onda, com hastes de 0,15 m, sendo uma com capacitor e uma sem capacitor no início da guia, com espaçamento de 0,009 m entre hastes e outra sem capacitor, com espaçamento de 0,022 m entre hastes. Essas guias de onda, juntamente com guias originais do fabricante com hastes de $0,20 \mathrm{~m}$, espaçadas $0,022 \mathrm{~m}$, foram inseridas individualmente em cada recipiente. Dados de umidade obtidos gravimetricamente e pelo analisador TRASE, e da constante dielétrica, foram tomados usando-se todas as guias de onda em cada recipiente durante a secagem do solo, de $0,35 \mathrm{~m}^{3} \mathrm{~m}^{-3}$ a $0,10 \mathrm{~m}^{3} \mathrm{~m}^{-3}$. Três modelos matemáticos foram ajustados aos dados de umidade e da correspondente constante dielétrica do solo gerados pelas guias de onda do fabricante. Um modelo exponencial foi considerado como mais adequado para estimativas dos teores de água, em função da constante dielétrica para a guia de onda do fabricante. Todas as guias de onda avaliadas apresentam viabilidade de uso, desde que previamente calibradas.
\end{abstract}

Palavras-chave: calibração, guia de onda, umidade, reflectometria no domínio do tempo

\section{Performance of different waveguides for use with the TRASE water content analyser}

\begin{abstract}
The objective of this work was to evaluate the relations between soil water content and the soil bulk dielectric constant, and to study different waveguides of a TRASE soil water content analyzer that operates according to TDR principles. Non-destructive and destructive samples of two sites of different texture of a Dystrophic Yellow Latossol were collected and packed into $10 \mathrm{~L}$ containers, resulting in four replications for each texture and structure. Three different waveguides were built with rods of 0.15 m length, one with a capacitor at the beginning of the waveguide, one without a capacitor, with rods $0.009 \mathrm{~m}$ apart, and another without a capacitor, with rods $0.022 \mathrm{~m}$ apart. These waveguides, together with buriable standard ones supplied by the manufactures with $0.20 \mathrm{~m}$ rods $0.022 \mathrm{~m}$ apart, were inserted in each sample. Soil water content was obtained by gravimetry and estimated by the TRASE analyzer, based on soil bulk dielectric constants using all waveguides during the soil drying process with water contents changing from $0.35 \mathrm{~m}^{3} \mathrm{~m}^{-3}$ to 0.10 $\mathrm{m}^{3} \mathrm{~m}^{-3}$. Three mathematical models were fitted to soil water content and bulk dielectric constant data. An exponential model was the most suitable in estimating soil water content as a function of bulk dielectric constant for the standard waveguide. All evaluated waveguides were feasible for use as long as they are previously calibrated.
\end{abstract}

Key words: calibration, waveguide, soil water content, time-domain-reflectometry

\section{INTRODUÇÃO}

A quantidade e a distribuição da umidade através do perfil do solo, são grandezas indispensáveis para estudos hidrológicos em áreas cultivadas. O uso racional da água, cada vez mais necessário na agricultura, está intimamente ligado às propriedades do sistema solo-água-planta, em que o conhecimento das propriedades desse sistema depende da informação do teor de água no solo, sendo que sua determinação pode ser efetuada por diversos métodos, como a 
gravimetria (Gardner, 1986) considerado método-padrão, e por métodos que estimam a umidade a partir de outras propriedades do solo, tais como termalização de nêutrons (Gardner, 1986), resistência elétrica (Spaans \& Baker, 1992), dissipação de calor (Phene et al., 1973), reflectometria no domínio do tempo (Topp et al., 1980) e tensiometria (Cassel \& Klute, 1986). Dentre essas metodologias, a técnica da reflectometria no domínio do tempo - TDR, é uma das mais vantajosas quanto ao uso, principalmente pela precisão, possibilidade de multiplicação de leituras, repetição sem destruição da amostra de solo e pela segurança (Topp et al., 1980; Or \& Wraith, 1997). O uso da reflectometria no domínio do tempo para determinação de teores de água do solo, tem sido limitado pelos custos, tanto do testador de cabos como das guias de onda; no último, existe ainda a limitação de uso a apenas uma profundidade, o que tem sido contornado com a disponibilização de hastes multiplicadoras, que permitem a leitura de teores de água a diferentes profundidades e por hastes segmentadas que, por diferença no diâmetro e material, permitem variação na impedância ao longo das mesmas, e leituras em diferentes profundidades no perfil do solo (Topp \& Davis, 1985; Conciani et al., 1997; Souza et al., 1999; Souza et al., 2000).

A determinação dos teores de água do solo pela técnica de TDR é feita a partir de valores da constante dielétrica aparente, correlacionados aos dados de teor de água, por meio de curvas de calibração obtidas a partir de dados de campo ou laboratório (Conciani et al., 1997). Os baixos valores da constante dielétrica do ar $\left(\varepsilon_{\mathrm{b}}=1\right)$, da matriz sólida $\left(\varepsilon_{\mathrm{b}}=3\right)$ comparados ao da água $\left(\varepsilon_{\mathrm{b}}=80\right)$ tornam o método pouco sensível à composição textural e à estrutura do solo (Or \& Wraith, 1997). Topp et al. (1980) propuseram um modelo polinomial de terceiro grau para estimativa dos teores de água:

$$
\theta_{\mathrm{v}}=-5,3 \times 10^{-2}+2,92 \times 10^{-2} \varepsilon_{\mathrm{b}}-5,5 \times 10^{-4} \varepsilon_{\mathrm{b}}^{2}+4,3 \times 10^{-6} \varepsilon_{\mathrm{b}}^{3}
$$

donde $\theta\left(\mathrm{m}^{3} \mathrm{~m}^{-3}\right)$ é o teor de água em base volumétrica e $\varepsilon_{\mathrm{b}}$ é a constante dielétrica aparente do solo (adimensional) que pode ser calculada conforme Or \& Wraith (1999):

$$
\varepsilon_{\mathrm{b}}=\left(\frac{\mathrm{c} \Delta \mathrm{t}}{2 \mathrm{~L}}\right)^{2}
$$

em que:

$\Delta \mathrm{t}$ - tempo de propagação $(\mathrm{T})$ da onda eletromagnética na haste da guia de onda

c - velocidade da luz $\left(\mathrm{L} \mathrm{T}^{-1}\right)$

L - comprimento da haste (L).

Este modelo tem sido usado por diversos autores (Baker \& Allmaras, 1990; Coelho \& Or, 1996; Souza et al., 1999) em monitoramento do estado da água do solo, para fins de estudo de transferências no sistema solo-planta-atmosfera.

O modelo de Topp et al. (1980) não tem embasamento físico, podendo não ser o mais adequado em todas as situações mais necessitando-se, portanto, de calibrações específicas, quando uma precisão maior das avaliações é requerida, principalmente em solos argilosos com baixos valores de densidade do solo
(Roth et al., 1992; Malicki et al., 1996). Outro modelo que tem sido utilizado para estimar o teor de água do solo com base na constante dielétrica aparente, é o modelo simplificado de Roth et al. (1990) desenvolvido com embasamento físico, considerando-se as fases sólida, líquida e gasosa do solo. O modelo de Roth et al. (1990) também conhecido por multifásico, é dado pela expressão:

$$
\theta_{\mathrm{v}}=\frac{\sqrt{\varepsilon_{\mathrm{b}}}-(2-\eta)}{8}
$$

em que:

$$
\eta \quad \text { - porosidade total do solo }\left(\mathrm{m}^{3} \mathrm{~m}^{-3}\right) \text {. }
$$

Este método gera uma curva de calibração semelhante à do modelo de Topp et al. (1980) para teores de água entre $0 \mathrm{e}$ $0,5 \mathrm{~m}^{3} \mathrm{~m}^{-3}$.

A literatura tem sido escassa em relação a informações sobre desenho de guias de onda para uso com testador de cabos. As guias de onda fornecidas por fabricantes de analisadores de umidade que funcionam com base em TDR, são de custo elevado, dificultando sua aquisição. Na sua construção, temse dado mais atenção ao espaçamento entre hastes (Petersen et al., 1995) que tem variado na prática, sem uma definição precisa, e em relação ao número de hastes, que também tem sido diversificado, indo desde duas até quatro (Zegelin et al., 1989).

Este trabalho teve por objetivos avaliar: (a) três modelos para estimativa do teor de água, em função da constante dielétrica de dois materiais de solo de tabuleiro costeiro; (b) o efeito da textura e da estrutura na constante dielétrica do solo e (c) a viabilidade de uso de três guias de onda apropriadas para o analisador de umidade Trase System I*.

\section{MATERIAL E MÉTODOS}

O experimento foi conduzido em casa-de-vegetação da Embrapa-Mandioca e Fruticultura, no período de 25/02 a 23/03 de 2000. Amostras de solo deformadas e indeformadas foram retiradas do campo em um Latossolo Amarelo Distrófico, em dois locais de solo de textura diferente (Tabela 1). As amostras deformadas foram destorroadas e secas ao ar sendo, em seguida, acondicionadas em recipientes de volume $10 \mathrm{~L}$, com altura de $0,23 \mathrm{~m}$ e diâmetro médio de $0,21 \mathrm{~m}$, enquanto as amostras indeformadas foram retiradas em recipientes semelhantes e levadas ao laboratório onde se fizeram os ajustes às dimensões dos recipientes. Usaram-se oito recipientes para amostras deformadas e oito para amostras não deformadas. A Tabela 1 apresenta as características granulométricas e físicas das amostras.

Os 16 recipientes foram dispostos sobre uma bancada numa casa-de-vegetação, procedendo-se, em seguida, à saturação do solo, para o que se determinou a densidade do solo (Blake \& Hartge, 1986a) e de partículas (Blake \& Hartge, 1986b) além da porosidade total (Danielson \& Sutherland, 1986). A diferença entre a porosidade total e o teor de água das amostras nos recipientes, indicou o volume necessário para saturação. 
Tabela 1. Caracterização física dos materiais de solo

\begin{tabular}{|c|c|c|c|c|c|c|c|}
\hline \multirow{2}{*}{$\begin{array}{l}\text { Classe } \\
\text { Textural }\end{array}$} & \multirow{2}{*}{ Estrutura } & Areia & Silte & Argila & \multicolumn{2}{|c|}{ Densidade $\left(\mathrm{Mg} \mathrm{m}^{-3}\right)$} & \multirow{2}{*}{$\begin{array}{l}\text { Porosidade Total } \\
\qquad\left(\mathrm{m}^{3} \mathrm{~m}^{-3}\right)\end{array}$} \\
\hline & & & $\left(\mathrm{g} \mathrm{kg}^{-1}\right)$ & & Solo & Partícular & \\
\hline \multirow{2}{*}{$\begin{array}{c}\text { Franco- } \\
\text { argiloso- } \\
\text { arenoso }\end{array}$} & Deformada & 590 & 95 & 315 & 1,39 & 2,50 & 0,44 \\
\hline & Indeformada & 590 & 95 & 315 & 1,33 & 2,50 & 0,47 \\
\hline \multirow{2}{*}{$\begin{array}{c}\text { Argiloso- } \\
\text { arenoso }\end{array}$} & Deformada & 589 & 17 & 394 & 1,48 & 2,61 & 0,43 \\
\hline & Indeformada & 589 & 17 & 394 & 1,47 & 2,61 & 0,44 \\
\hline
\end{tabular}

Em cada recipiente contendo solo de estrutura deformada ou indeformada, foi inserida uma guia de onda original do fabricante, com três hastes de $0,20 \mathrm{~m}$ de comprimento e separadas $0,025 \mathrm{~m}$. Nos quatro recipientes contendo o solo franco-argiloarenoso, foram inseridas, próximo às guias de onda originais, três guias de onda contínuas, construídas artesanalmente. Uma guia de onda foi feita com haste de aço inoxidável, com $0,15 \mathrm{~m}$ de comprimento e $0,003 \mathrm{~m}$ de diâmetro, espaçadas $0,009 \mathrm{~m}$ contendo, no início e ligando duas antenas, um capacitor de 4 PiF (Andrade \& Or, sd). Uma outra guia de onda foi feita da mesma forma da anterior, exceto por não conter capacitor no início das hastes, e uma terceira foi também construída sem capacitor, mas com espaçamento entre hastes, de $0,025 \mathrm{~m}$.

Após a instalação das antenas com o solo saturado, os recipientes foram mantidos com a superfície do solo exposta, para permitir perda de água apenas por evaporação. Quando a média das umidades nos baldes atingiu $0,35 \mathrm{~m}^{3} \mathrm{~m}^{-3}$, deu-se início à coleta de dados dos teores de água do solo, que foram determinados por pesagens diárias dos recipientes durante $o$ período de 27 dias, até atingirem $0,10 \mathrm{~m}^{3} \mathrm{~m}^{-3}$, durante o qual a temperatura média do ar na casa-de-vegetação foi de $33,2^{\circ} \mathrm{C}$ e a umidade relativa média foi de $54 \%$. Foram feitas leituras diárias da constante dielétrica aparente do solo, $\varepsilon_{\mathrm{b}}$, e umidade pela tela de medida direta do analisador Trase, para a guia de onda original do fabricante e para a guia de onda com capacitor. A constante dielétrica aparente do solo também foi estimada para todas as guias de onda, por meio de leituras manuais obtidas da tela de gráfico, determinando-se o tempo de propagação da onda eletromagnética, $\Delta \mathrm{t}$ (Eq. 2) na haste.

Quando os teores de água atingiram valores próximo a $0,10 \mathrm{~m}^{3} \mathrm{~m}^{-3}$, retiraram-se três amostras coletadas em pontos diferentes do recipiente, de modo a representar todo o volume de solo, previamente conhecido, para determinação do seu peso seco. Com base no peso seco da amostra, determinou-se o peso seco de todo o volume de solo contido no recipiente e, pelas pesagens diárias (peso úmido) determinaram-se os teores de água correspondentes a cada pesagem.

As análises constaram de: (i) aferição do analisador Trase, feita com os valores de $\varepsilon_{\mathrm{b}}$ medidos pelas guias de onda originais, obtidos diretamente do aparelho e pela leitura do tempo $(\Delta \mathrm{t})$ na tela de gráfico do instrumento; (ii) avaliação de diferentes modelos de ajuste da umidade, como função de $\varepsilon_{\mathrm{b}}$; (iii) avaliação de $\varepsilon_{\mathrm{b}}$ nos diferentes materiais de solo, com respeito à textura $\mathrm{e}$ estrutura e (iv) avaliação das guias de onda construídas com base nos valores de $\varepsilon_{\mathrm{b}}$ e das umidades obtidas com essas guias. As análises foram baseadas em ajustes de modelos lineares e não lineares aos dados de umidade, como função de $\varepsilon_{\mathrm{b}}$, e em ajustes de modelos lineares aos dados de $\varepsilon_{\mathrm{b}}$ obtidos com uso das guias originais, como função de $\varepsilon_{\mathrm{b}}$, obtidos com uso das guias construídas. Além dos modelos de Topp et al. (1980) e de
Roth et al. (1990) para representar os dados de umidade como função da constante dielétrica aparente do solo, diversos modelos foram também ajustados aos dados, pelo aplicativo "Table curve 2D". Computaram-se desvios percentuais entre as variáveis, como umidade ou constante dielétrica aparente, por exemplo, para dada variável, como umidade obtida por gravimetria $\left(\theta_{\mathrm{gi}}\right)$ e, pela leitura no analisador Trase $\left(\theta_{\mathrm{ci}}\right)$ determinou-se o desvio percentual $\left(\mathrm{Dp}_{\mathrm{i}}\right)$ entre os dois valores, pela equação:

$$
\mathrm{DP}_{\mathrm{i}}=\frac{\left(\theta_{\mathrm{gi}}-\theta_{\mathrm{ci}}\right) \cdot 100}{\theta_{\mathrm{gi}}}
$$

A média e o desvio-padrão da média desses desvios percentuais calculados para uma variável, foram utilizados nas análises propostas.

O efeito do tipo de solo e do estado estrutural das amostras nos valores da constante dielétrica aparente do solo, foi avaliado por meio das análises de sensibilidade (equação 4) e de variância, para um delineamento inteiramente casualizado, com fatorial 2 (tipos de solo) x 2 (estrutura deformada e indeformada) x 4 repetições.

\section{RESULTADOS E DISCUSSÃO}

\section{Aferição do analisador de umidade}

Na Figura 1 está ilustrada a relação entre as constantes dielétricas aparentes $\left(\varepsilon_{\mathrm{b}}\right)$ medidas pelas diferenças entre os tempos de propagação dos pulsos eletromagnéticos $(\Delta \mathrm{t})$ e lidas diretamente no visor do aparelho para os dois tipos de solo

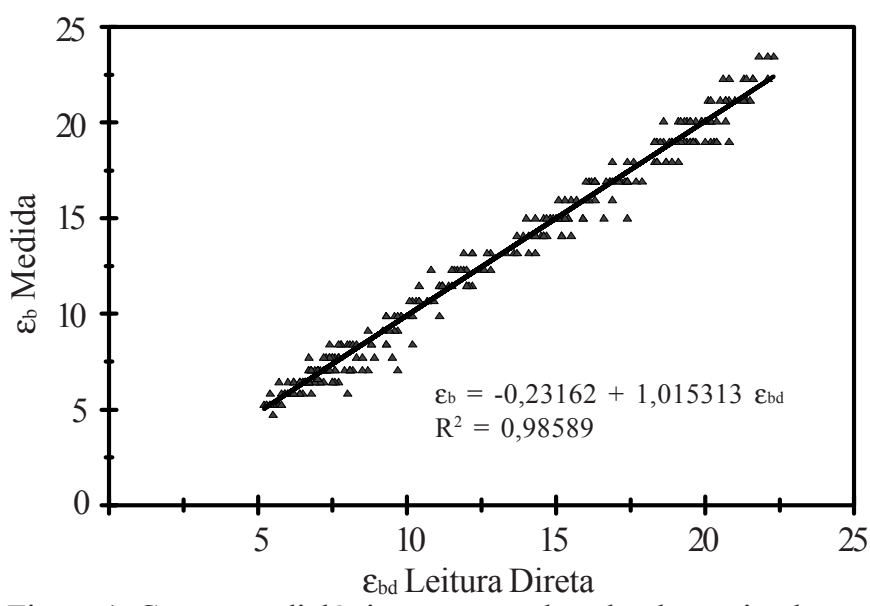

Figura 1. Constante dielétrica aparente do solo, determinada por meio de $\Delta$ t obtido na tela de gráfico $\left(\varepsilon_{\mathrm{b}}\right)$, em função da constante lida diretamente na tela de medida do analisador Trase $\left(\varepsilon_{\mathrm{bd}}\right)$ 
(amostras deformadas e indeformadas). Existem diversos registros diferentes de $\varepsilon_{\mathrm{b}}$ lidos diretamente no aparelho para um mesmo valor medido de $\Delta \mathrm{t}$, o que se deve à maior sensibilidade do "software" do instrumento, quanto à definição do início e do final da guia de onda, comparado à leitura na tela de gráfico do instrumento; apesar dessas diferenças, a média dos desvios entre a $\varepsilon_{\mathrm{b}}$ lida e a medida pelos valores de $\Delta \mathrm{t}$ foi de $3,2 \%$, com desvio-padrão dessa média de $4,2 \%$, considerados baixos. $\mathrm{O}$ teste $\mathrm{t}$ não apresentou diferença significativa entre as médias das $\varepsilon_{\mathrm{b}}$ a $5 \%$ de probabilidade. Esses resultados demonstram confiabilidade na aferição do instrumento.

\section{Teor de água $x$ constante dielétrica aparente do solo}

Na Figura 2 são comparados os modelos de estimativa do teor de água do solo, obtidos por gravimetria e a partir dos valores de $\varepsilon_{\mathrm{b}}$ alcançados com uso das guias de onda originais (do fabricante). No caso dos diversos modelos testados para ajuste aos dados de teores de água em base volumétrica, o modelo exponencial do tipo $\theta=\mathrm{a}+\mathrm{b} \exp \left[\varepsilon_{\mathrm{b}} / \mathrm{c}\right]$, com $\mathrm{a}=0,24809097, \mathrm{~b}=0,27055556$ e $\mathrm{c}=29,36014$, foi o de melhor ajuste aos dados de teor de água em todos os casos observados, isto é, para os dois tipos e para os dois estados estruturais de solo. O modelo de Topp et al. (1980) e o simplificado de Roth et al. (1990) apresentaram pequena diferença dentro da faixa de teores de água avaliada e relativa distância dos teores de água medidos gravimetricamente, principalmente entre $0,15 \mathrm{~m}^{3} \mathrm{~m}^{-3} \mathrm{e}$ 0,32 $\mathrm{m}^{3} \mathrm{~m}^{-3}$. A Tabela 2 apresenta alguns parâmetros para avaliação da dispersão entre os teores de água estimados pelos três modelos e medidos por gravimetria.

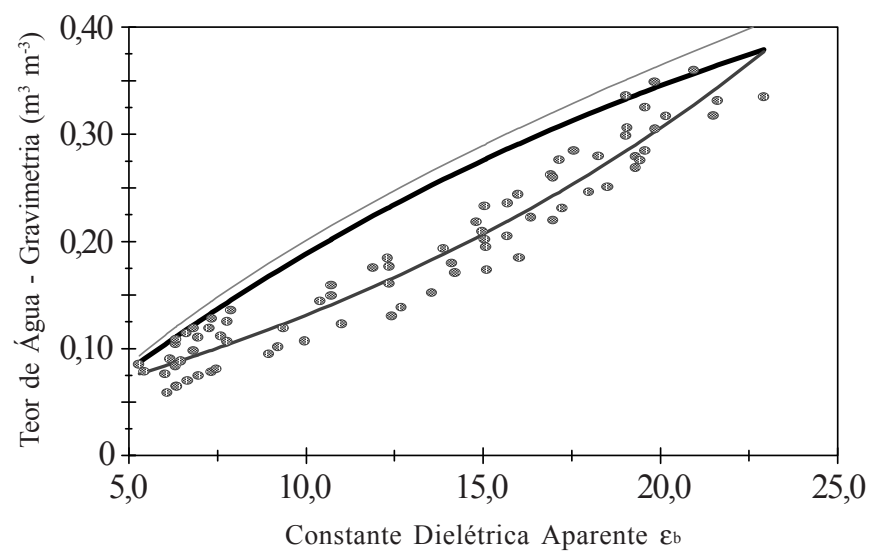

- Topp et al. (1980) — Exponencial $\quad$ observado - Roth et al. (1990)

Figura 2. Ajustes de diferentes modelos aos dados de teor de água do solo, em função da constante dielétrica, considerando-se as texturas franco-argilo-arenosa e argilo-arenosa e as estruturas deformada e não deformada

Tabela 2. Análise de sensibilidade relativa aos desvios entre as estimativas dos teores de água obtidas pelos modelos de Topp et al. (1980), Roth et al. (1990) e exponencial e as medidas dos teores de água por gravimetria

\begin{tabular}{cccccc}
\hline \multirow{2}{*}{ Modelo } & $\begin{array}{c}\text { Média dos } \\
\text { Desvios }\end{array}$ & $\begin{array}{c}\text { Desvio } \\
\text { Padrão }\end{array}$ & Teste ${ }^{*}$ & $\mathrm{R}^{2}$ \\
\cline { 2 - 3 } & \multicolumn{2}{c}{$\%$} & & \\
\hline Topp et al. (1980) & 31,52 & 23,14 & $\mathrm{p}<0,05$ & 0,5790 \\
Roth et al. (1990) & 39,87 & 25,10 & $\mathrm{p}<0,05$ & 0,3675 \\
Exponencial & 4,40 & 3,03 & $\mathrm{p}>0,05$ & 0,9373 \\
\hline Modelo - gravimetria & & & & \\
\hline
\end{tabular}

O modelo simplificado de Roth et al. (1990) apresentou a mais alta média dos desvios entre os valores estimados e os valores de umidade medidos, e o menor coeficiente de determinação. O modelo de Topp et al. (1980) embora com menor média dos desvios e menor desvio-padrão em relação ao modelo de Roth et al. (1990) resultou num coeficiente de determinação bem inferior ao do modelo exponencial. O teste $t$ para comparação das médias entre os valores de umidade estimados pelos dois modelos e os valores medidos, foi significativo para os modelos de Topp et al. (1980) e de Roth et al. (1990) indicando diferença entre as médias obtidas por esses modelos e a média dos teores de água medidos. Não houve diferença significativa entre as estimativas resultantes do modelo exponencial e os teores de água medidos, em que o modelo exponencial foi o de melhor ajuste aos teores de água medidos, com desvio médio de $4,4 \%$ e coeficiente de determinação de 0,9373. Esses resultados não podem ser generalizados, uma vez que divergem, em parte, dos obtidos por Tommaselli (1997) que obteve o modelo polinomial cúbico como superior a todos os outros modelos, apresentando os maiores valores de coeficiente de correlação para solos com textura fina. O mesmo autor verificou, entretanto, que o termo cúbico de $\theta$ deixa de ser importante à medida que a textura do solo se torna mais grossa.

\section{Efeito do tipo e da estrutura do solo}

$\mathrm{Na}$ Tabela 3 observa-se o quadro de análise de variância, que permite avaliar-se o efeito da textura e estrutura do solo, na obtenção dos valores de $\varepsilon_{\mathrm{b}}$ pelo analisador Trase, para as guias de onda originais. Pela Tabela, verifica-se que o teste $F$ foi não significativo, indicando não ter havido diferença entre as médias das constantes dielétricas aparentes dos tratamentos (textura e estrutura do solo). Considerando-se os mesmos valores de teor de água do solo em valores absolutos da constante dielétrica aparente, houve maior diferença entre as médias relativas aos dois tipos de solo que entre as médias relativas aos dois estados estruturais do solo (Tabela 4). Dados da Tabela 1 mostram que os valores da porosidade total em todos os tratamentos foram muito próximos e, assim, as proporções do volume de solo ocupado pela matriz sólida e por espaço vazio para ar ou água, aproximaram-se. É de se esperar pequena influência da textura e da estrutura do solo na determinação dos teores de água pela TDR, pelos baixos valores das constantes dielétricas das fases sólida e gasosa do solo, comparados aos da água. Desta forma, as diferenças observadas na Tabela 1, tanto para densidade do solo como para densidade de partículas, foram insuficientes para causar diferenças relevantes nos valores da $\varepsilon_{\mathrm{b}}$. Esses resultados confirmam a maior sensibilidade do analisador de umidade, com base nos princípios da reflectometria no domínio do tempo à presença de água, o que tornou a calibração pouco sensível dentro das condições de estudo.

Tabela 3. Análise de variância do experimento em delineamento inteiramente casualizado, fatorial 2 x 2, com quatro repetições

\begin{tabular}{lcccc}
\multicolumn{1}{c}{$\begin{array}{c}\text { Fontes de } \\
\text { Variação }\end{array}$} & G.L. $\begin{array}{c}\text { Soma de } \\
\text { Quadrados }\end{array}$ & $\begin{array}{c}\text { Quadrado } \\
\text { Médio }\end{array}$ & F \\
\hline Solo & 1 & 0,03116 & 0,03116 & $0,108(\mathrm{p}>0,05)$ \\
Estrutura & 1 & 0,62910 & 0,62910 & $2,175(\mathrm{p}>0,05)$ \\
Solo x Estrutura & 1 & 0,31165 & 0,31165 & $0,108(\mathrm{p}>0,05)$ \\
Resíduo & 12 & 3,47167 & 0,28930 & \\
\hline Coeficiente de variacão $=3,20 \%$ & & &
\end{tabular}


Tabela 4. Comparação dos desvios entre os valores das constantes dielétricas aparente, referentes aos dois tipos de solo e aos dois estados estruturais

\begin{tabular}{lrr}
\hline \multirow{2}{*}{ Tratamentos } & $\begin{array}{c}\text { Média dos } \\
\text { Desvios }\end{array}$ & $\begin{array}{c}\text { Desvio } \\
\text { Padrão }\end{array}$ \\
\cline { 2 - 3 } & \multicolumn{2}{c}{$(\%)$} \\
\hline Franco-argilo-arenoso x argilo-arenoso & 12,30 & 12,38 \\
Deformada x indeformada & 5,67 & 4,05 \\
\hline
\end{tabular}

(A)

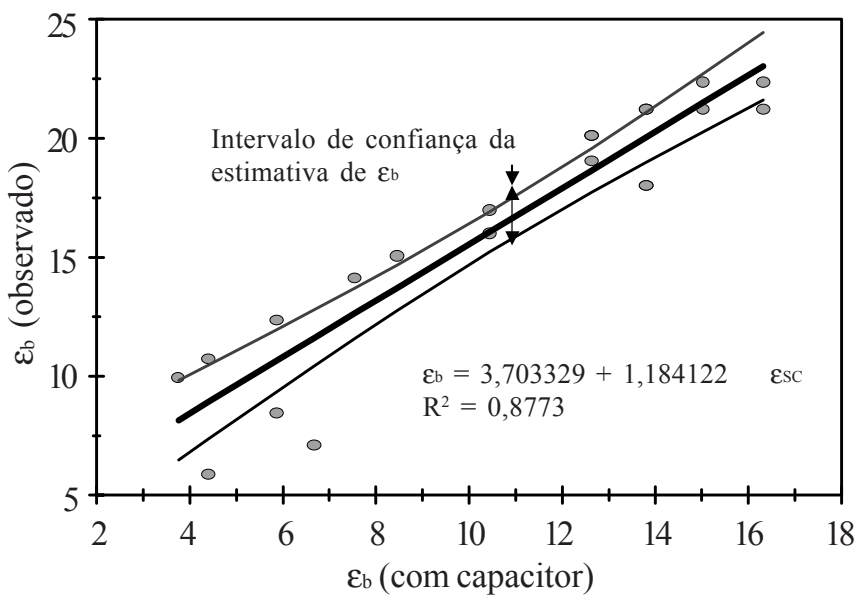

(B)

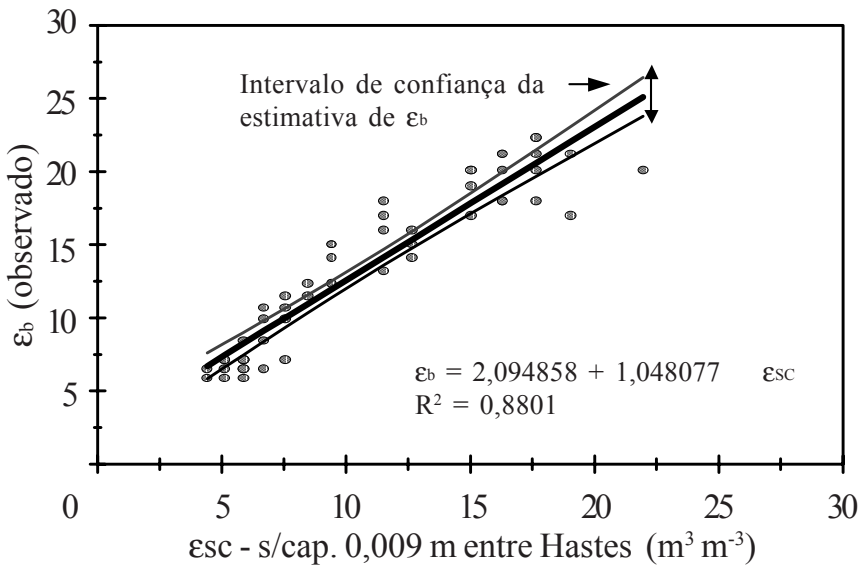

(C)

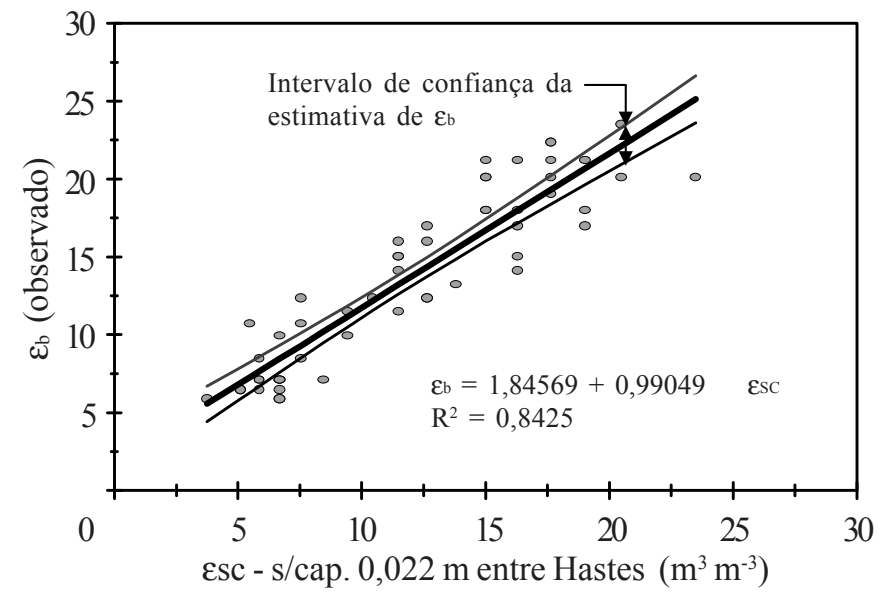

Desempenho das guias de onda construídas em laboratório

Os valores de $\varepsilon_{\mathrm{b}}$ e do teor de água gerados pelas guias de onda construídas, quando comparados aos valores medidos com as guias de onda originais, não resultaram em uma função linear do tipo $\mathrm{y}=\mathrm{x}$, mas permitiram ajuste de funções lineares do tipo $y=a+b x$. A Figura $3(A, B$ e C) ilustra os ajustes dessas funções aos valores de $\varepsilon_{\mathrm{b}}$ obtidos com uso dessas guias de onda, em relação aos valores encontrados com as guias originais
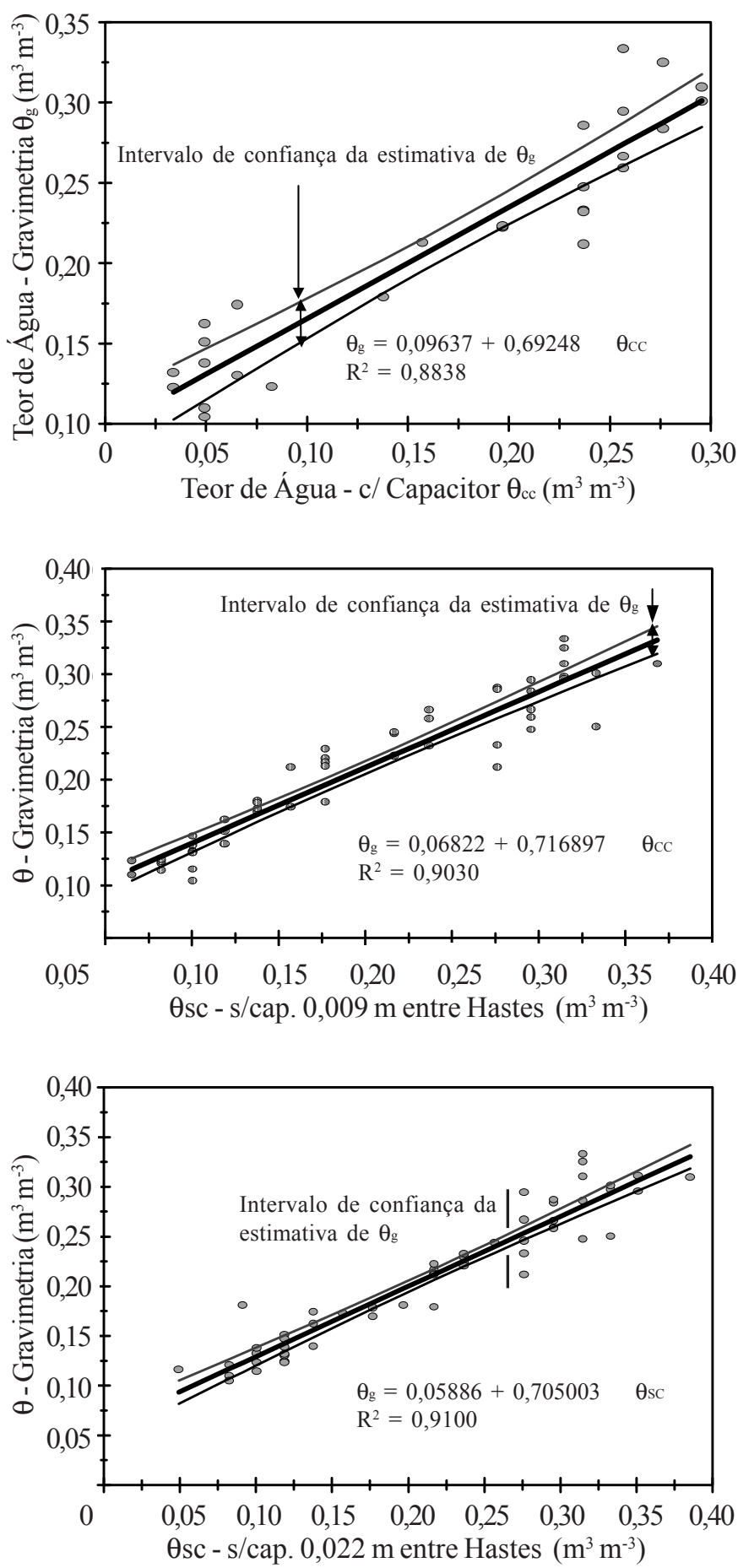

Figura 3. Calibração da constante dielétrica aparente do solo pela guia de onda do fabricante, $\varepsilon_{\mathrm{b},}$, teor de água obtido por gravimetria, $\theta_{\mathrm{g}}$, em função da constante dielétrica, e (A) teor de água $\left(\theta_{\mathrm{cc}}, \varepsilon_{\mathrm{cc}}\right)$ obtidos com a guia de onda construída com capacitor no início das hastes, (B) teor de água $\left(\theta_{\mathrm{sc}}, \varepsilon_{\mathrm{sc}}\right)$ obtidos com a guia de onda construída sem capacitor no início das hastes, espaçadas $0,009 \mathrm{~m}$ e (C) teor de água $\left(\theta_{\mathrm{sc}}, \varepsilon_{\mathrm{sc}}\right)$ obtidos com a guia de onda construída sem capacitor no início das hastes, espaçadas $0,022 \mathrm{~m}$ 
do fabricante, e dos teores de água obtidos com uso dessas guias e por gravimetria. As diferenças entre os coeficientes de determinação dos ajustes para os dados de teor de água não foram suficientes para destacar as guias de onda por seu desempenho. Os intervalos de confiança das estimativas da variável dependente dos modelos lineares, foram maiores para as guias de onda com capacitor no início das hastes, sendo que a média dos desvios entre os teores de água calculados usando essas guias e os teores de água determinados por gravimetria, foi de 10,2\%, com desvio-padrão de 7,3\%. As guias de onda sem capacitor no início das hastes, espaçadas $0,009 \mathrm{~m}$, quando relacionadas às guias originais, resultaram em melhores ajustes dos modelos lineares aos dados de $\varepsilon_{\mathrm{b}}$ e de teores de água do solo, com os intervalos de confiança mais estreitos que os gerados pelas guias com capacitor. A média dos desvios entre os teores de água gerados por essas guias e os teores de água determinados por gravimetria, foi de $8,1 \%$, com desviopadrão de $6,5 \%$.

As guias de onda sem capacitor no início das hastes, espaçadas $0,022 \mathrm{~m}$, resultaram em um ajuste muito próximo do obtido para as guias sem capacitor, com hastes espaçadas 0,009 $\mathrm{m}$, tanto para $\varepsilon_{\mathrm{b}}$ como para os teores de água do solo. A média dos desvios entre os teores de água gerados por essas guias e os teores de água determinados por gravimetria $(7,5 \%)$ bem como os desvios-padrão da média $(7,2 \%)$, reforçam a proximidade do desempenho dos dois tipos de guia de onda. As guias com hastes espaçadas $0,022 \mathrm{~m}$ seguiram as mesmas dimensões das guias originais do fabricante, exceto pelo artifício de detecção do início da haste.

As guias sem capacitores apresentaram maior precisão de uso em comparação com as guias com capacitor no início das hastes, sendo que não houve diferença, em termos práticos, entre seu desempenho. As guias com capacitores apresentam vantagem sobre as guias sem capacitores, quanto à operacionalidade nas leituras, isto é, as primeiras permitem leituras diretas, usando somente a tela de medida do analisador Trase, enquanto as sem capacitores requerem o uso da tela de gráfico, onde o operador é quem define o início e o final da haste da guia. Os resultados indicaram que os três tipos de guia de onda podem ser usados para medição do teor de água do solo, com a ressalva de que os valores devem ser corrigidos pelos modelos de regressão apresentados e para outros tipos de solo; tais modelos devem ser previamente ajustados para o uso dessas guias.

\section{CONCLUSÕES}

1. O modelo exponencial foi o mais adequado para se estimar teores de água em função da constante dielétrica dos dois tipos e estrutura dos solos estudados.

2. Os valores da constante dielétrica aparente do solo não foram afetados pelas variações, tanto no tipo de solo (textura) como na sua estrutura (deformada e indeformada).

3. As guias de onda sem capacitor com espaçamento entre hastes de $0,009 \mathrm{~m}$, apresentaram desempenho semelhante às com espaçamento entre hastes de $0,022 \mathrm{~m}$ na estimativa do teor de água, embora com o uso de diferentes modelos de regressão.
4. Não houve diferenças entre o desempenho das guias de onda com capacitor e sem capacitor.

\section{LITERATURA CITADA}

Andrade, C. de L.T. de; Or, D. Desenvolvimento e calibração de guias de onda para TDR tipo TRASE. Sete Lagoas: Embrapa Milho e Sorgo, 2000 (comunicação pessoal).

Baker, J.M.; Allmaras, R.R. System for automating and multiplexing soil moisture measurement by time-domain reflectometry. Soil Science Society of America Journal. Madison, v.54, p.1-6, 1990.

Blake, G.R.; Hartge, K.H. Bulk density. In: Klute, A. (ed.) Methods of soil analysis. Madison, Wisconsin: American Society of Agronomy. 1986a. p.374-390.

Blake, G.R.; Hartge, K.H. Particle density. In: Klute, A. (ed.) Methods of soil analysis. Madison, Wisconsin: American Society of Agronomy. 1986b. p.371-373.

Cassel, D.K.; A. Klute. Water potential: Tensiometry. In: Klute, A. (ed.) Methods of Soil Analysis. Madison, Wisconsin: American Society of Agronomy. 1986. p.563-596.

Conciani, W.; Carneiro, B.J.; Soares, M.M.; Hermann, P.S.P.; Crestana, S. Emprego de TDR com sondas multihastes segmentadas para medida de umidade de um perfil de solo. In: Simpósio Nacional de Instrumentação Agropecuária, 1, 1997, São Carlos, Anais... São Carlos. EMBRAPA-CNPDIA. p.169-173, 1997.

Coelho, E.F.; OR, D. A parametric model for two-dimensional water uptake by corn roots under drip irrigation. Soil Science Society of America Journal. Madison, v.60, p.1039-1049.1996.

Danielson, R.E.; Sutherland, P.L. Porosity. In: Methods of soil analysis. Klute, A. (ed.) p.443-462. Madison, Wisconsin: American Society of Agronomy. 1986.

Gardner, W.H. Water content. In: Methods of soil analysis. Ed. A. Klute, Madison, Wisconsin: American Society of Agronomy. p.493-544. 1986.

Malicki, M.A.; Plagge, R.; Roth, C.H. Imporoving the calibration of dieletric TDR soil moisture determination taking into account the solid soil. European Journal of Soil Science, v.47, p.357-366, 1996.

Or, D.; Dasberg, S. Drip irrigation. Nova York: Spriger-Verlag. 1999. 162p.

Or, D.; Wraith, J.M. Agricultural and environmental soil physics. Logan, Utah: Utah State University. 1997. 218p.

Petersen, L.W.; Thomsen, A.; Moldrup, P.; Jacobsen, O.H.; Rolston, D.E. High-resolution time domain reflectometry: sensitivity dependency on probe design. Soil Science. Baltimor, v.159, n.3.p.149-155. 1995.

Phene, C.J.; Hoffman, G.J.; Austin, R.S. Controlling automated irrigation with soil matric potential sensor. Transactions of the ASAE, St. Joseph, v.16, p.773-776. 1973.

Roth, C.H.; Malicki, M.A.; Plagge, R. Empirical evaluation of relationship between soil dieletric constant and volumetric water content as the basis for calibration of moisture measurements by TDR. Journal of Soil Science. Reading, v.43, p.1-13, 1992. 
Roth, C.H.; Schulin, R.; Fluhler, H.; Attinger, W. Calibration of time-domain reflectometry for water content measurement using composite dieletric approach. Water Resources Research, Washington, v.26, n.10, p.2267-2273. 1990.

Souza, C.F.; Matsura, E.E.; Testezlaf, R. Desempenho de sondas multihaste segmentada para o monitoramento da umidade do solo por meio da técnica de TDR. In: Congresso Brasileiro de Engenharia Agrícola, 28, 1999, Pelotas:SBEA/UFPel, 1999.

Souza, C.F.; Matsura, E.E.; Testezlaf, R. Avaliação de sonda de TDR multihaste segmentada na determinação do bulbo molhado em irrigação localizada. In: Congresso Brasileiro de Engenharia Agrícola, 29, 2000, Ceará: SBEA/UFC, 2000.

Spaans, E.J.A.; Baker, J. Calibration of watermark soil moisture sensors for soil matric potential and temperature. Plant and Soil, .v.143, p.213-217. 1992.
Tommaselli, J.T.G. Influência de algumas características do solo sobre a calibração de um aparelho de TDR (Time Domain Reflectometry). Piracicaba: CENA/USP. 1997,167p. Tese Doutorado

Topp, G.C.; Davis, J.L.; Annan, A. Electromagnetic determination of soil water content: Measurement in coaxial transmission lines. Water Resources Research, Washington, v.16, p.576583, 1980.

Topp, G.C.; Davis, J.L. Measurement of soil water content using time domain reflectometry (TDR): A field evaluation. Soil Science Society of America Journal, Madison, v.49, p.19-24, 1985.

Zegelin, S.J.; White, I.; Jenkins, D.R. Improved field probes for soil water content and electrical conductivity measurements using time domain reflectometry. Water Resources Research, Washington, v.25, n.11, p.2367-2376, 1989. 